\section{Prioritizing and stopping}

\section{prescription medicines}

$\mathrm{P}$ harmaceuticals have had a remarkable effect on improving life expectancy and quality of life. However, most medicines need not be used lifelong, and the ongoing benefits of specific drugs must be considered in the clinical and social contexts in which they are prescribed. Pressing reasons remain for doctors and patients to rigorously reconsider which medications are really needed (prioritization) and which medications could be stopped (discontinuation).

Reasons for prioritization and discontinuation are many and well documented, yet simple heuristics to guide clinicians are rarely articulated. Prioritization and discontinuation of medications become important, for example, when a patient approaches the end of life and goals that were formerly curative become palliative. ${ }^{1}$ A considerable proportion of prescriptions incur outof-pocket costs that are burdensome to many patients. Simplification of patients' regimens improves adherence. Inappropriate use of medications and polypharmacy are known to be widespread. ${ }^{2}$ Yet, ethical and clinical uncertainty about the indefinite use of some therapies such as antidementia drugs makes discontinuation a key point of discussion: the only way to assess if a medicine is still needed may be to stop the medication.

At times, both patients and physicians prioritize different treatments by forgoing some beneficial remedies in favour of others perceived as being more valuable. For example, patients may prioritize medicines according to out-ofpocket prescription costs, or personal $\sim$ beliefs about safety or effectiveness. Side effects also influence patients' adherence. Because physicians are often unaware of patients' prioritization efforts, they are often unable to assist patients to make decisions about the relative importance of different medicines.
Physicians are also forced to prioritize different treatments, as the number and complexity of tradeoffs between different treatment options increases. For example, individual drugs may be efficacious and recommended by guidelines and yet not make as much sense when considered with other relevant treatment recommendations for comorbid conditions. Differing goals of care, patient preference and benefits (e.g., frequency and magnitude of benefit, time to attain it) versus burdens of various treatments (e.g., the frequency and magnitude of adverse effects, adherence burden, costs) are all worthy of consideration.

Several barriers can retard efforts to stop medicines. The act of writing or renewing a prescription summarizes and carries forth a relationship between the physician and patient, and may be perceived as an expression of caring and concern. Physicians and patients are both vulnerable to a bias toward the status quo; once a corrective is prescribed, they may hesitate to discontinue it. ${ }^{3}$ Some barriers to discontinuation involve economic and other incentives: Pharmaceuticals are heavily advertised and marketed, whereas no counterpart exists to promote discontinuation. The so-called prescribing cascade, in which one agent is prescribed to control for the side effects of another, also accumulates medications, with little or no countervailing force toward discontinuation. ${ }^{2}$ Finally, professional barriers also prevent efforts to prioritize and discontinue medications; for example, a patient may see a specialist and be prescribed a new medicine with unclear directions (to the patient or to fellow health practitioners) about length of use.

The choice of which drugs are the most important to continue and which can be safely removed should ideally be evidence-based, but there are few data on the safety and optimal means of discontinuing most medicines. Most studies that have examined discontinuation of therapeutics have focused on a few specific classes or types (e.g., anticonvulsants, psychoactive drugs and diuretics) or on select populations (such as elderly or nursing-home patients). The range of contexts and methods used in these studies is exhibited in the Appendix to this article (available at www.cmaj.ca/cgi/content /full/174/8/1083/DC1).

"N-of-1" clinical trials, in which the principles of large randomized trials are applied to treatment choices for a single patient, are also relevant to medication discontinuation. However, these trials are generally used to compare efficacy rather than to assess whether a therapy is needed at all. $N$-of- 1 trials are also hampered by practical costs and complexity (a well-controlled $N$-of-1 trial may require the assistance of a pharmacist to ensure double-blinding, can take time and can be expensive). Despite these limitations, the principle of comparing 2 treatment options within an individual patient may be particularly useful in elderly patients. An $\mathrm{N}$-of-1 trial can help a practitioner to avoid many well-described biases that interfere with interpretation of the safety and effectiveness of a given drug; a disease's natural history may, for example, may lead to improvements that occur in spite of, rather than because of, a specific therapy.

Prioritization and discontinuation can be improved (Table I) with careful physician attention. At the outset, there are often discrepancies between medical records and patients' actual use of prescriptions, so any effort to prioritize or discontinue medications has to begin with a review of what the patient is using. This review should extend beyond written prescriptions to include common nonprescription medicines, dietary supplements and herbal remedies. This review may identify duplicate therapies, prescription errors or drugs that could safely be used on an as-needed basis rather than taken each day (e.g., a proton-pump inhibitor for mild gastroesophageal reflux disease).

For many common conditions such 
Table 1: Opportunities for prioritization or discontinuation of medication

Degree of uncertainty

and reason for action

Examples: a patient who. .

None or minimal (low stakes)

To correct $\quad$ Is taking 2 therapeutically equivalent versions of a single drug

a medical error $\quad$ Is elderly and taking propoxyphene

- Has a history of angioedema from an ACE inhibitor that has not been stopped

To simplify regimen - Is taking a thiazide diuretic and an ACE inhibitor that can be changed to a single combined preparation

Clinical benefit $\quad$ Is a young man with adequate diet taking a multivitamin

is unlikely - Was recently prescribed long-term magnesium because of a mildly decreased value discovered incidentally on a laboratory test

Safe for use on - Has mild-to-moderate arthritis taking analgesics such as a Cox-2 inhibitor

an as-needed basis - Has reflux taking a gastroprotective agent such as a proton-pump inhibitor

- Has seasonal allergies using daily anti-histamines or decongestants

Moderate (moderate stakes) - a discontinuation trial is appropriate because:

Benefit has likely - Is taking a selective serotonin-reuptake inhibitor for 12 months after a first episode of depression

been achieved - Has used anticonvulsants for 24 months after a first episode of generalized tonic-clonic seizures without identifiable cause

- Is using estrogen replacement therapy for menopausal symptoms

A behavioural - Has not tried diet and exercise but is taking a statin for elevated cholesterol

intervention can - Takes anxiolytics for insomnia but is considering behavioural interventions instead

be substituted

- Uses an anticholinergic for urinary incontinence but is willing to try a trial of timed voiding

Benefit is unlikely - Has a life expectancy of 1 year and is taking a bisphosphonate for osteopenia

to be realized

- Is frail, has severe emphysema with cor pulmonale and is taking a statin for primary prevention of cardiac events

High (high stakes)

Careful prioritization - Has multiple symptomatic conditions, is taking 15 medicines, has persistent problems with memory and is necessary organization, and has subjective complaints of being on too many medicines

Note: $\mathrm{ACE}$ = angiotensin-converting enzyme.

as depression, idiopathic generalized seizure or menopause, therapy discontinuation may be appropriate after a specified period. Discontinuation may also be appropriate when lifestyle modification and behavioral intervention can replace pharmacotherapy, or when the benefit of intervention is unlikely to be realized in time. ${ }^{1}$

Patients' values and preferences are important to consider throughout this model. However, a model of shared decision-making is particularly important in settings with greater uncertainty or risk (Table I).

Considerably more scientific research goes into studying when to start medicines than into when to stop them. Nevertheless, medication prioritization and discontinuation can decrease costs, simplify prescription regimens, decrease risks of adverse drug events and polypharmacy, focus thera- pies where they are most effective, and prevent cost-related underuse of medications. Many questions remain unanswered, such as how to reconcile multiple clinical guidelines that have been developed in isolation, and how to determine where the most benefit can be gained from medication discontinuation in specific patients. With the growing elderly component of our population, rising prescription expenditures and the expanding variety of pharmaceuticals available to treat chronic diseases, the answers to these questions are increasingly important.

\section{G. Caleb Alexander}

Robert Wood Johnson Clinical

Scholars Program

MacLean Center for Clinical Medical

Ethics

Section of General Internal Medicine Department of Medicine

\section{Maliha A. Sayla}

Section of General Internal Medicine Department of Medicine

Holly M. Holmes

Greg A. Sachs

Section of Geriatrics

Department of Medicine

University of Chicago

Chicago, Ill.

Competing interests: None declared.

Acknowledgements: We thank Ken Covinsky, Donald Redelmeier and anonymous reviewers for their helpful comments on drafts of this manuscript.

\section{REFERENCES}

I. Holmes HM, Cox-Hayley D, Alexander GC, et al. Reconsidering medication appropriateness for patients late in life. Arch Intern Med 2006; 66 . In press.

2. Rochon PA, Gerwitz JH. Optimising drug treatment for elderly people: the prescribing cascade. BMJ I997;315:1096-9.

3. Redelmeier D, Rozin P, Kahneman D. Understanding patients' decisions: cognitive and emotional perspectives. JAMA I993;270:72-6. 